\title{
Asymptotic model for velocity dip position in open channels
}

\author{
Snehasis Kundu ${ }^{1}$
}

Received: 27 March 2017/ Accepted: 4 July 2017/Published online: 11 July 2017

(c) The Author(s) 2017. This article is an open access publication

\begin{abstract}
An empirical model is proposed to predict the velocity dip position at the central section of open channels. The model is fitted based on asymptotic matching technique and validated by using a wide range of aspect ratios (channel width/flow depth) from 0.155 to 15 . The matching approach, which relies on dividing the trend into smaller segments that can be combined into an overall relation, employs regression technique and thus warrants the best-fit accuracy results. The obtained model satisfies the upper and lower bounds of dip positions equal to 0.5 and 1 , respectively. A comparison with other formulas widely reported in the literature is provided. The model is also applied to predict Reynolds shear stress and velocity distribution in open channels. This model will help extending our ability for analyzing velocity field in open channels under different flow and boundary conditions.
\end{abstract}

Keywords Velocity distribution - Secondary current . Asymptotic matching $\cdot$ Aspect ratio $\cdot$ Sidewall effect
RMSE Root mean square error (-)

$s_{1} \quad$ Sum of squared relative error (-)

$\mathrm{s}_{2} \quad$ Sum of logarithmic deviation error $(-)$

$u \quad$ Mean longitudinal velocity $(\mathrm{m} / \mathrm{s})$

$u^{\prime} \quad$ Longitudinal velocity fluctuation $(\mathrm{m} / \mathrm{s})$

$u^{*} \quad$ Shear velocity $(\mathrm{m} / \mathrm{s})$

$v^{\prime} \quad$ Vertical velocity fluctuation $(\mathrm{m} / \mathrm{s})$

$x \quad$ Longitudinal direction (m)

$y \quad$ Vertical direction (m)

$y_{\mathrm{d}} \quad$ Location of dip position (m)

$z \quad$ Lateral direction (m)

$Y_{\mathrm{i}} \quad$ Asymptotes (-)

$\lambda, \beta \quad$ Parameters (-)

$\kappa \quad$ Von Karman coefficient (-)

$\Pi \quad$ Coles' wake parameter (-)

$\xi_{d} \quad$ Dimensionless dip position (-)

$\xi_{0} \quad$ Zero velocity bed level (m)

$\xi_{d, c} \quad$ Computed value of $\xi_{d}(-)$

$\xi_{d, o} \quad$ Observed value of $\xi_{d}(-)$

$\varphi_{i} \quad$ Matching functions (-)

\author{
Abbreviations \\ Ar $\quad$ Aspect ratio (-) \\ $b \quad$ Channel width (m) \\ $b_{0}, b_{1} \quad$ Parameters (-) \\ $h \quad$ Flow depth (m) \\ $m \quad$ Matching constant (-) \\ MASE Mean absolute standard error (-) \\ $N \quad$ Total number of data points (-) \\ $r \quad$ Average percentage relative error (-)
}

Snehasis Kundu snehasis18386@gmail.com

1 Department of Basic Sciences and Humanities, IIIT Bhubaneswar, Bhubaneswar, Odisha 751003, India

\section{Introduction}

The vertical water velocity distribution in open channels is considered three-dimensional due to the presence of largescale circular secondary currents. In many cases, the location of the maximum streamwise velocity appears below the free surface (Francis 1878; Stearns 1883; Murphy 1904; Gibson 1909; Vanoni 1946; Nezu and Nakagawa 1993). An accurate estimation of the velocity dip position with respect to channel bottom is required for many practical applications including the assessment of sediment

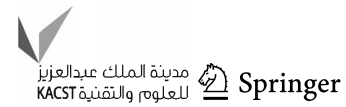


transport rates (Einstein 1950) and contamination (Guo and Julien 2008), and the precise definition of hydrodynamic flow conditions in urban drainage sewers (Lee and Julien 2006; Guo et al. 2015).

Nezu and Rodi (1985) found that the velocity dip position in open channels is related to the aspect ratio Ar, which is the ratio of channel width $b$ to water depth $h$. It is evident that at the central section of open channels the velocity dip phenomenon occurs when sidewall shear stresses dominate for $\mathrm{Ar}<5$. However, when $\mathrm{Ar}>5$, channels exhibit no velocity dip, i.e. the maximum water velocity appears at the top surface (Stearns 1883; Montes and Ippen 1973).

Predicting the velocity dip position at the central section of open channels within a wide range of aspect ratios is a difficult task. Several researchers provided empirical models to predict it. Based on data obtained from nine literatures, Wang et al. (2001) proposed an empirical relation applicable to narrow channels. Yang et al. (2004) proposed a model valid for aspect ratios from 4.1 to 15 showing that even for large aspect ratios the velocity dip occurs very close to the channel sidewall region. Bonakdari et al. (2008) showed that both models overestimate experimental data when the aspect ratio is small and suggested a new relation. Later, Guo (2013) studied vertical water velocity profiles in smooth rectangular channels and proved that the velocity dip position decreases exponentially from the water surface to the middle depth, as the aspect ratio is varied from infinity to zero. Guo (2013) suggested that the centreline vertical shear stress distribution is expressed as

$\tau=\tau_{0}\left(1-\xi_{d}\right)-\tau_{1} \xi$

where $\tau_{1}=\alpha \tau_{0}$ is called the 'apparent' shear stress at the free surface. Like Reynolds shear stress, $\tau_{1}$ only functions like a shear stress although it is essentially a momentum transfer by secondary currents near the water surface (Guo 2013). The parameter $\alpha$ has the range $0 \leq \alpha \leq 1$. If the condition $\tau=0$ at $\xi=\xi_{d}$, is imposed into Eq. (1), and from the bounds of parameter $\alpha$, one can obtain that $0.5 \leq \xi_{d} \leq 1$. He also found that the two models, besides that of Bonakdari et al. (2008), do not satisfy the following boundary conditions: $\xi_{\mathrm{d}} \rightarrow 0.5$ when $\mathrm{Ar} \rightarrow 0$ and $\xi_{\mathrm{d}} \rightarrow 1$ when $\mathrm{Ar} \rightarrow \infty$, where $\xi_{\mathrm{d}}$ denotes dimensionless distance of dip-position from channel bed. Pu (2013) proposed an empirical model for smooth and rough channel beds. Only those models for Guo (2013) and $\mathrm{Pu}$ (2013) satisfied asymptotically the abovementioned boundary conditions. Apart from these empirical model, some researchers suggested numerical methods and entropy based models to predict it. Guo and Julien (2008) envisaged that near the free surface, velocity profile is likely parabolic type and therefore a parabola can be fitted to find the dip-position.
Recently, Kundu (2017) suggested an entropy based approach to predict the dip-phenomena over entire cross section of open channels. Most of these previous models are tested with limited number of data sets and, therefore, may not produce good results for other data sets. Therefore, a more appropriate model is required for a wide range of data sets. In this study, we develop an empirical model based on the technique of asymptotic matching method proposed by Almedeij (2008, 2009, 2010) with a large number of data sets. The method has been applied to model the shields diagram and drag coefficient for large Reynolds number and has proven to provide good results.

This study suggests a new empirical formula to compute the velocity dip position in open channels. The formula will be validated by using field and experimental data covering a wide range of aspect ratios and compared with models found in the literature. A detailed accuracy analysis will be conducted to evaluate the results. The best applicability of the model will be discussed in estimating parameters for prediction of Reynolds shear stress and mean velocity distribution in open channels.

\section{Model development}

\section{Asymptotic matching technique by Almedeij (2008, 2009, 2010)}

A model for velocity dip position at central section of open channels can be developed by using asymptotic matching technique. The approach is based on dividing the possible entire range of the dip-position data into smaller segments not necessarily of same length. In each of the formed region, straight lines are fitted by linear regression, and then the segments for each region are combined together asymptotically into an overall relation (Almedeij 2008, 2009, 2010).

The matching procedure is described in Fig. 1a. In this matching method two possibilities may arise. These two cases are presented with simple schematic diagrams, each composed of linear segments $Y_{1}$ and $Y_{2}$ where each of them is expressed as $Y=b_{0} X+b_{1}$; where $b_{0}$ and $b_{1}$ are slope and intercept, respectively. For the case on the left side figure, the $b_{0}$ value increases from $Y_{1}$ to $Y_{2}$, and these two segments can be considered to have Monotone Increasing Slopes (MIS) with respect to $x$-axis. Similarly, for the other case on the right side figure the $b_{0}$ value decreases from $Y_{1}$ to $Y_{2}$, and hence these segments have Monotone decreasing slopes (MDS). For the case of MIS, Almedeij (2008) suggested that the linear asymptotes can be match asymptotically using the function $\varphi=Y_{1}^{m}+Y_{2}^{m}$, with the entire relation being $Y=(\varphi)^{1 / m}$; where $m$ is a matching constant. If $m$ value is large enough, then this expression 
Fig. 1 Schematic diagram of asymptotic matching method. Solid line represents the final model and the dashed lines represent the asymptotes with linear segments: a a series of two segments of $Y_{1}$ and $Y_{2}$ with the dash-dot lines being the entire relation of $Y_{1}$ and $Y_{2}$ fitted by using the corresponding exponent $m ; \mathbf{b}$ a series of four linear segments of monotone increasing slopes (MIS)
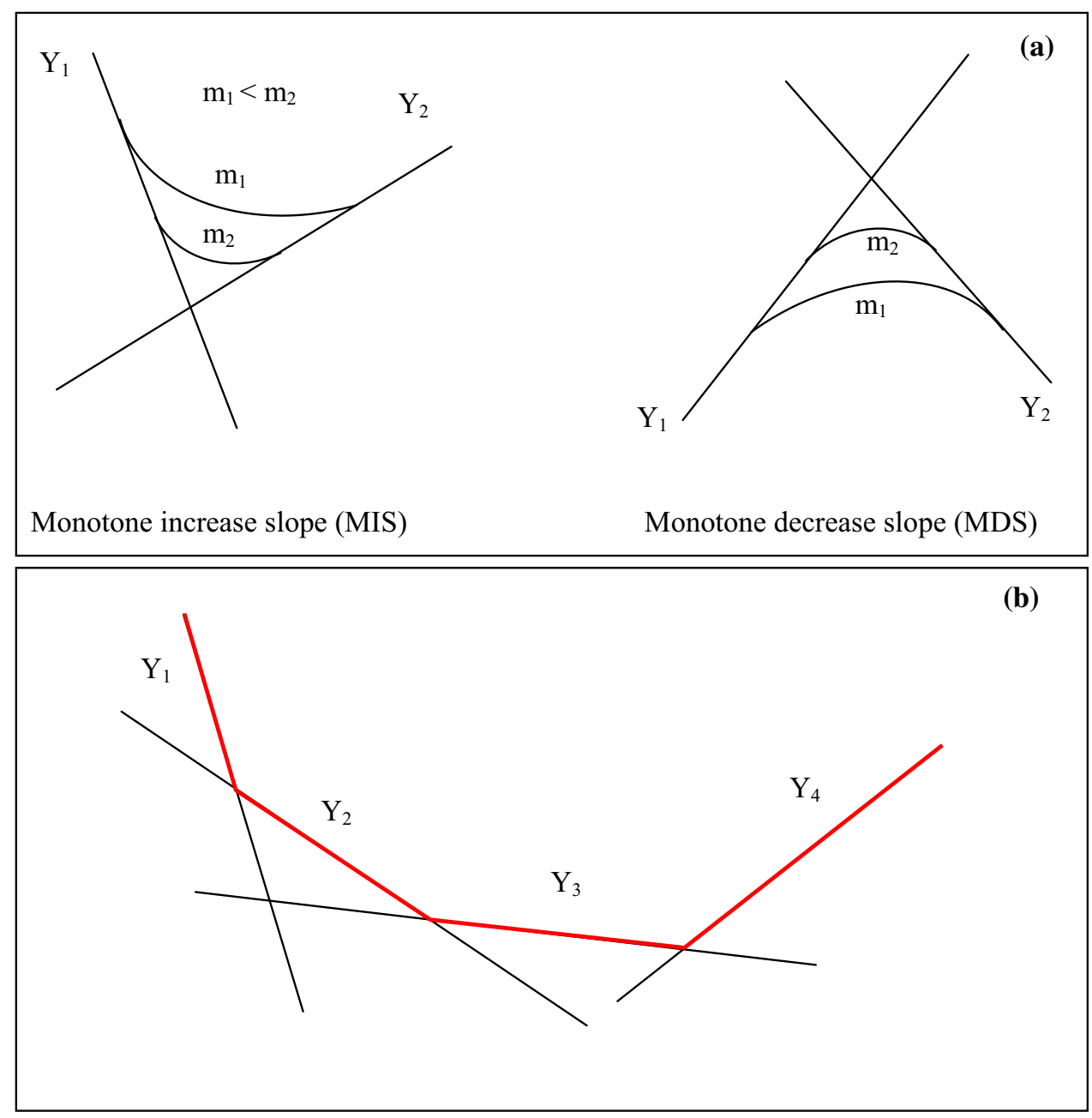

will automatically match the upper parts of the intersected asymptotes of $Y_{1}$ and $Y_{2}$ within the corresponding ranges of $X$. In general, the larger the $m$ value, the smoother is the matching at the joints of the segments. However, the value of $\varphi$ becomes excessively large for a very large $m$ value that cannot be calculated numerically, and the model fails to work. Similarly, for the MDS, the matching attempt is attained rather by the function $\varphi=1 /\left(Y_{1}^{-m}+Y_{2}^{-m}\right)$ with $Y=(\varphi)^{1 / m}$. Similarly, for large values of $m$, this expression will satisfy the lower parts of the intersected asymptotes.

For data sets having a large range, it can be divided into small parts. In each parts, the above procedure can be applied repeatedly. An example for a series of four segments of MIS is shown in Fig. 1b. In general, a series of segments of MIS is collected by the function

$\varphi=Y_{1}^{m}+Y_{2}^{m}+\cdots+Y_{k}^{m}$

and for MDS by

$\varphi=\frac{1}{Y_{1}^{-m}+Y_{2}^{-m}+\cdots+Y_{k}^{-m}}$ where $k$ is the total number of segments used in the series. For more details readers can go through Almedeij (2008, 2009, 2010).

\section{Proposed model}

Twenty six datasets obtained from the literature are considered here to examine the distribution pattern of velocity dip position in open channels. Details of data are given in Table 1, which shows that the range of aspect ratios is from 0.155 to 15 . For channel with oval shape (sewer shape), the aspect ratio is calculated by taking the ratio of width of the free surface to flow depth (for Larrarte (2006) data set). Figure 2 plots the relationship between the aspect ratio and velocity dip position. Lower and upper boundary limits are apparent for dip positions equal to 0.5 and 1 , respectively. In between, dip position data increase as aspect ratio becomes larger. It is worth noting that the data near the lower and upper boundaries match the limits asymptotically. The existence of the lower and upper limits has also 
Table 1 Details of selected datasets in steady and uniform flow

\begin{tabular}{|c|c|c|c|c|c|c|c|}
\hline No. & Source & Type & Channel cross section & Bed roughness & Flow depth $(\mathrm{cm})$ & Aspect ratio & Data no. \\
\hline 1 & Murphy (1904) & Experimental & Rectangular & Smooth & $178.2-285.2$ & $0.155-1.35$ & 5 \\
\hline 2 & Gibson (1909) & Experimental & Rectangular & Smooth & $14-57.3$ & $0.256-2$ & 5 \\
\hline 3 & Vanoni (1946) & Experimental & Rectangular & Rough & $7.1-16.9$ & $4.997-11.895$ & 28 \\
\hline 4 & NHRI (1957) & Experimental & Rectangular & Smooth & $8-29.27$ & $4.1-15$ & 24 \\
\hline 5 & Guy et al. (1966) & Experimental & Rectangular & - & $61-243.84$ & $7.94-8.54$ & 6 \\
\hline 6 & Rajaratnam and Muralidhar (1969) & Experimental & Rectangular & Smooth & - & $3.283-10.839$ & 4 \\
\hline 7 & Montes and Ippen (1973) & Experimental & Rectangular & Smooth & - & $5.505-7.656$ & 15 \\
\hline 8 & Knight and Macdonald (1979) & Experimental & Rectangular & Rough & - & $1.012-1.012$ & 1 \\
\hline 9 & Sarma et al. (1983) & Experimental & Rectangular & Smooth & $3.8-30.5$ & $2.024-7.984$ & 3 \\
\hline 10 & Zippe and Graf (1983) & Experimental & Rectangular & Rough & $12.3-16.8$ & $6.173-7.634$ & 5 \\
\hline 11 & Hu (1985) & Experimental & Rectangular & Rough & - & $1.664-11.132$ & 13 \\
\hline 12 & Nezu and Rodi (1985) & Experimental & Rectangular & Smooth & $10-60$ & $1.009-6.001$ & 3 \\
\hline 13 & Coleman (1986) & Experimental & Rectangular & Smooth & $16.7-17.2$ & $2.058-2.132$ & 7 \\
\hline 14 & Nezu and Rodi (1986) & Experimental & Rectangular & Smooth & $3.6-10.5$ & $0.189-5.42$ & 68 \\
\hline 15 & Cardoso et al. (1989) & Experimental & Rectangular & Smooth & $27.2-42.8$ & $4.673-7.353$ & 5 \\
\hline 16 & Wang and Qian (1989) & Experimental & Rectangular & Smooth & $8-10$ & $3-3.75$ & 3 \\
\hline 17 & Tominaga et al. (1989) & Experimental & Rectangular & Smooth & $2.5-10$ & $2.005-7.995$ & 3 \\
\hline 18 & Wang and Fu (1991) & Experimental & Rectangular & - & - & $4.166-4.291$ & 4 \\
\hline 19 & Kironoto and Graf (1994) & Experimental & Rectangular & Rough & $8.7-28.9$ & $2.069-6.897$ & 8 \\
\hline 20 & Song and Graf (1994) & Experimental & Rectangular & Rough & $13.08-20$ & $3.004-4.588$ & 12 \\
\hline 21 & Wang and An (1994) & Experimental & Rectangular & Rough & $7.2-7.8$ & $3.797-4.167$ & 5 \\
\hline 22 & Yang (1996) & Experimental & Rectangular & Smooth & 11 & $4.47-9.84$ & 88 \\
\hline 23 & Graf and Cellino (2002) & Experimental & Rectangular & Smooth & 12 & 5 & 17 \\
\hline 24 & Larrarte (2006) & Field & Oval with a bench & Smooth & $56-122$ & $1.756-2.925$ & 25 \\
\hline 25 & Ghoshal (2004) & Experimental & Rectangular & Rough & 35 & 1.43 & 1 \\
\hline 26 & Yan et al. (2011) & Experimental & Rectangular & Smooth & $6-18$ & $2.306-6.999$ & 5 \\
\hline
\end{tabular}

been observed by others such as the experimental work of $\mathrm{Hu}$ and Hui (1995).

Figure 2 also provides possible linear segments of $Y_{1}, Y_{2}$ and $Y_{3}$ that can be used to match the velocity dip position data. The asymptotes $Y_{1}$ and $Y_{3}$ were chosen to satisfy the lower and upper boundary conditions. From the selected data in Table 1, the highest value of aspect ratio is obtained as 15 . From the pattern of data in Fig. 4, one can observe that for $\mathrm{Ar}>10$, dip-position vanishes which indicates that after this value, dip-position becomes independent of aspect ratio of channel. Therefore, the highest value of aspect ratio is chosen as 15 as from data. This value is chosen as fixed for the present study. Initially, the entire range of aspect ratios from 0 to 15 is divided into two regions of $0<\mathrm{Ar}<5$ and $5<\mathrm{Ar}<15$. In the first region, data are fitted by two linear segments as $Y_{1}=0.48$ and $Y_{2}=a_{0} A r+b_{0}$, where $a_{0}(>0)$ and $b_{0}$ are constants to be determined form data. The asymptote $Y_{1}$ is chosen in such a way that the final developed model satisfies the lower asymptotic boundary condition. In the second region, data are fitted with only one linear segment as $Y_{3}=1$. The segments $Y_{1}$ and $Y_{2}$ can be combined by using the expression $\varphi_{1}=\left(Y_{1}^{m}+Y_{2}^{m}\right)^{1 / m}$ for MIS. Both $\varphi_{1}$ and $Y_{3}$ can then be combined by using $\varphi=\left[1 /\left(\varphi_{1}^{-m}+Y_{3}^{-m}\right)\right]^{1 / m}$ for MDS where $m$ is the matching constant. Combining all the asymptotes, the overall model becomes

$\xi_{\mathrm{d}}=\varphi=\left[\frac{1}{\left\{0.48^{m}+\left(a_{0} A r+b_{0}\right)^{m}\right\}^{-1}+1}\right]^{1 / m}$

where $\xi_{\mathrm{d}}\left(=y_{\mathrm{d}} / h\right)$ is dimensionless distance of dip position from channel bed, and $y_{\mathrm{d}}$ denotes distance of dip position from channel bed. The values of the parameters $a_{0}(>0)$ and $b_{0}$ and $m$ are obtained from the least square method in MATLAB and they are given as $a_{0}=0.0895, b_{0}=0.4718$ and $m=16.09$. The coefficient of regression $R^{2}$ for the model is obtained as 0.85 . The low value of coefficient of regression is due to the fluctuation of the data points for $\mathrm{Ar}$ $\leq 8$. The fluctuation of data may occur due to measurement error, change of roughness of the bottom boundary and due to change of bed elevation at the central section. 
Fig. 2 The asymptotes and the final model

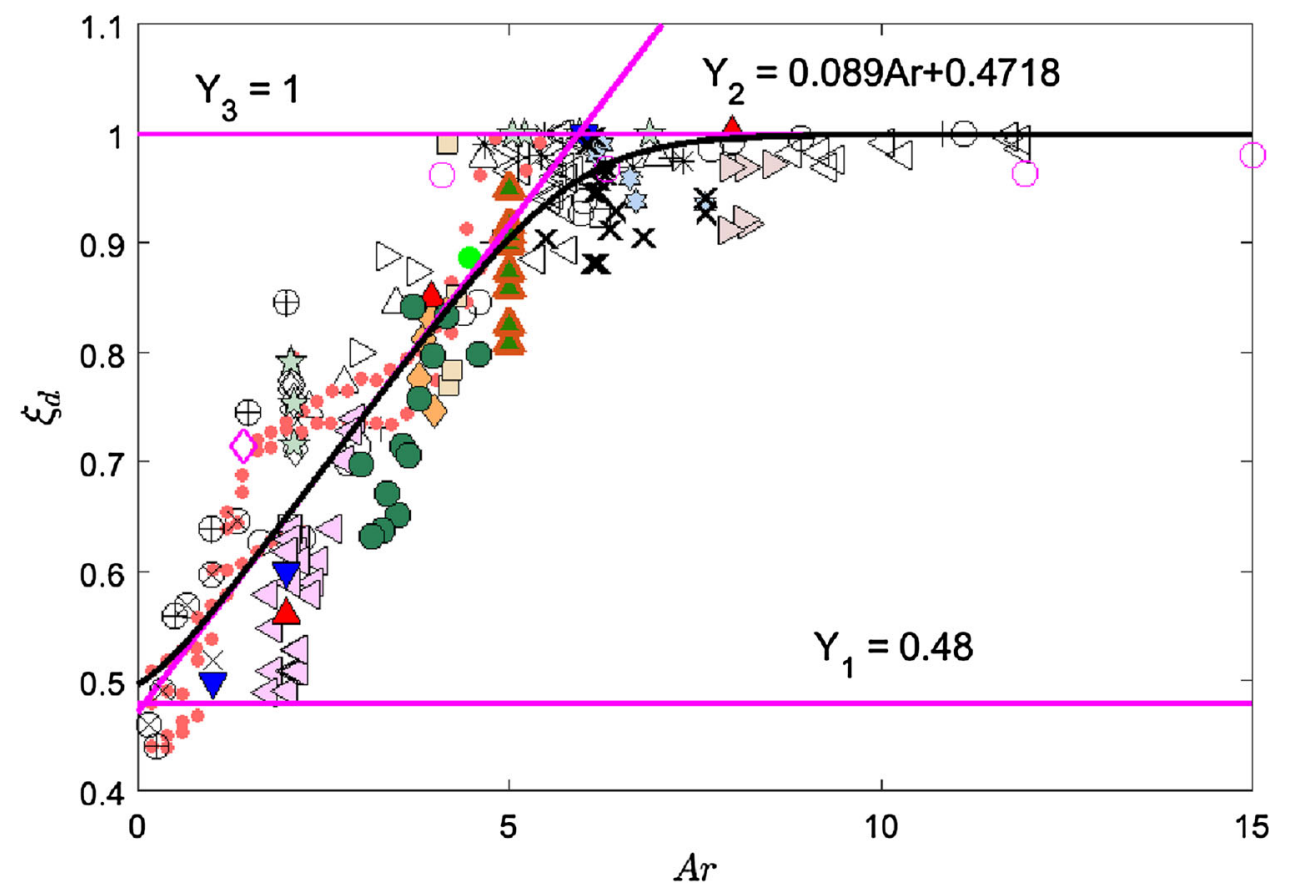

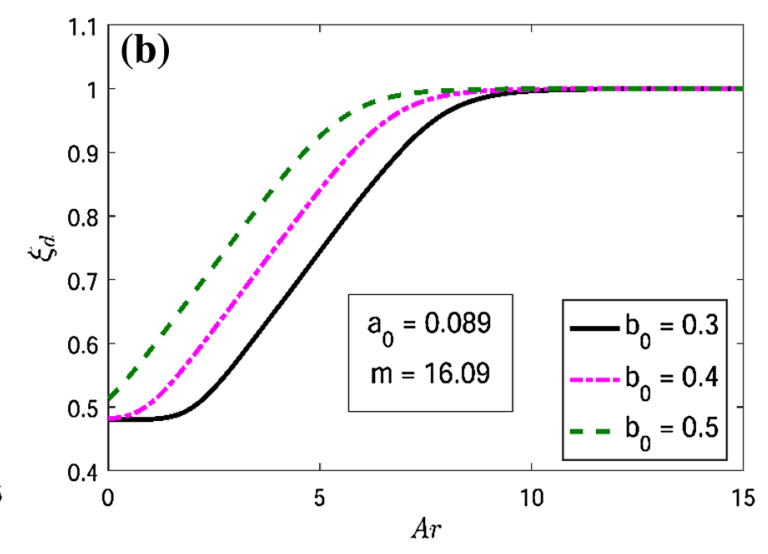

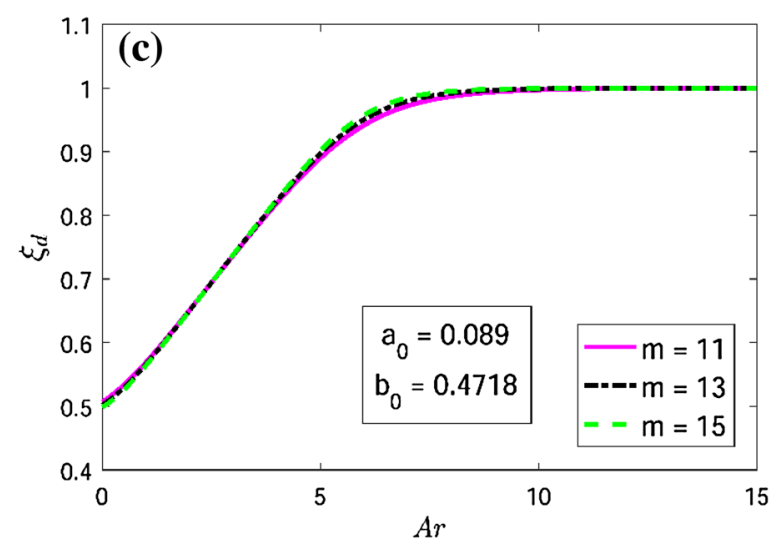

Fig. 3 Variation of model with parameters

It can be observed from the model that it contains three parameters $a_{0}(>0)$ and $b_{0}$ and $m$. The variations of the model with these are parameters are shown in Fig. 3. In
Fig. 3a the variation with $a_{0}$ for four different values of it are shown. Similarly in Fig. 3b and in Fig. $3 c$ the variation for three different values of $b_{0}$ and $m$ are shown 
respectively. From the figure it can be seen that model is very sensitive for $a_{0}$ and $b_{0}$ and comparatively less sensitive for the parameter $m$. Optimizing the values of parameters, the above-mentioned values are obtained for the best effective result.

\section{Comparison with other formulas}

The model can be compared with other empirical formulas reported in the literature. The formulas are those of Wang et al. (2001), Yang et al. (2004), Bonakdari et al. (2008), Guo (2013) and $\mathrm{Pu}$ (2013). Table 2 shows the mathematical expressions for the models. The model of Wang et al. (2001), which is applicable to narrow open channels, has a sinusoidal wave component. The models of Bonakdari et al. (2008) and Pu (2013), applicable to wide and narrow open channels, are of sigmoid type. The model of $\mathrm{Pu}$ (2013) is a modification of Bonakdari et al. (2008) for rather rough boundaries. The models of Yang et al. (2004) and Guo (2013), applicable to both wide and narrow open channels, are of exponential decay.

All models are presented graphically in Fig. 4. From the figure, it can be seen that the models provide nearly similar patterns. However, only Eq. (4), Guo (2013) and Pu (2013) satisfy the lower and upper bounds.

To assess the models' goodness of fit in an objective manner, the following statistical parameters are chosen: mean absolute standard error (MASE), average percentage relative error $(r \%)$, sum of squared relative error $\left(s_{1}\right)$, sum of logarithmic deviation error $\left(s_{2}\right)$, and root mean square error (RMSE). These statistics are expressed correspondingly as,

$\operatorname{MASE}=\frac{1}{N} \sum_{i=1}^{N} M_{i}$

$M_{i}= \begin{cases}\xi_{\mathrm{d}, \mathrm{c}} / \xi_{\mathrm{d}, \mathrm{o}} & \text { if } \xi_{\mathrm{d}, \mathrm{c}}>\xi_{\mathrm{d}, \mathrm{o}} \\ \xi_{\mathrm{d}, \mathrm{o}} / \xi_{\mathrm{d}, \mathrm{c}} & \text { if } \xi_{\mathrm{d}, \mathrm{o}}>\xi_{\mathrm{d}, \mathrm{c}}\end{cases}$

$r=\frac{1}{N} \sum_{i=1}^{N} \frac{\left|\xi_{\mathrm{d}, \mathrm{c}}-\xi_{\mathrm{d}, \mathrm{o}}\right|}{\xi_{\mathrm{d}, \mathrm{o}}} \times 100(\%)$

$s_{1}=\sum_{i=1}^{N} \frac{\left(\xi_{\mathrm{d}, \mathrm{c}}-\xi_{\mathrm{d}, \mathrm{o}}\right)^{2}}{\xi_{\mathrm{d}, \mathrm{o}}^{2}}$

$s_{2}=\sum_{i=1}^{N}\left(\log \left|\xi_{\mathrm{d}, \mathrm{c}}\right|-\log \left|\xi_{\mathrm{d}, \mathrm{o}}\right|\right)^{2}$

$\operatorname{RMSE}=\sqrt{\frac{1}{N} \sum_{i=1}^{N}\left(\xi_{\mathrm{d}, \mathrm{c}}-\xi_{\mathrm{d}, \mathrm{o}}\right)^{2}}$

where $N$ denotes the total number of data points, and $\xi_{\mathrm{d}, \mathrm{c}}$ and $\xi_{\mathrm{d}, \mathrm{o}}$ denote the computed and observed values of dimensionless velocity dip position, respectively. The 


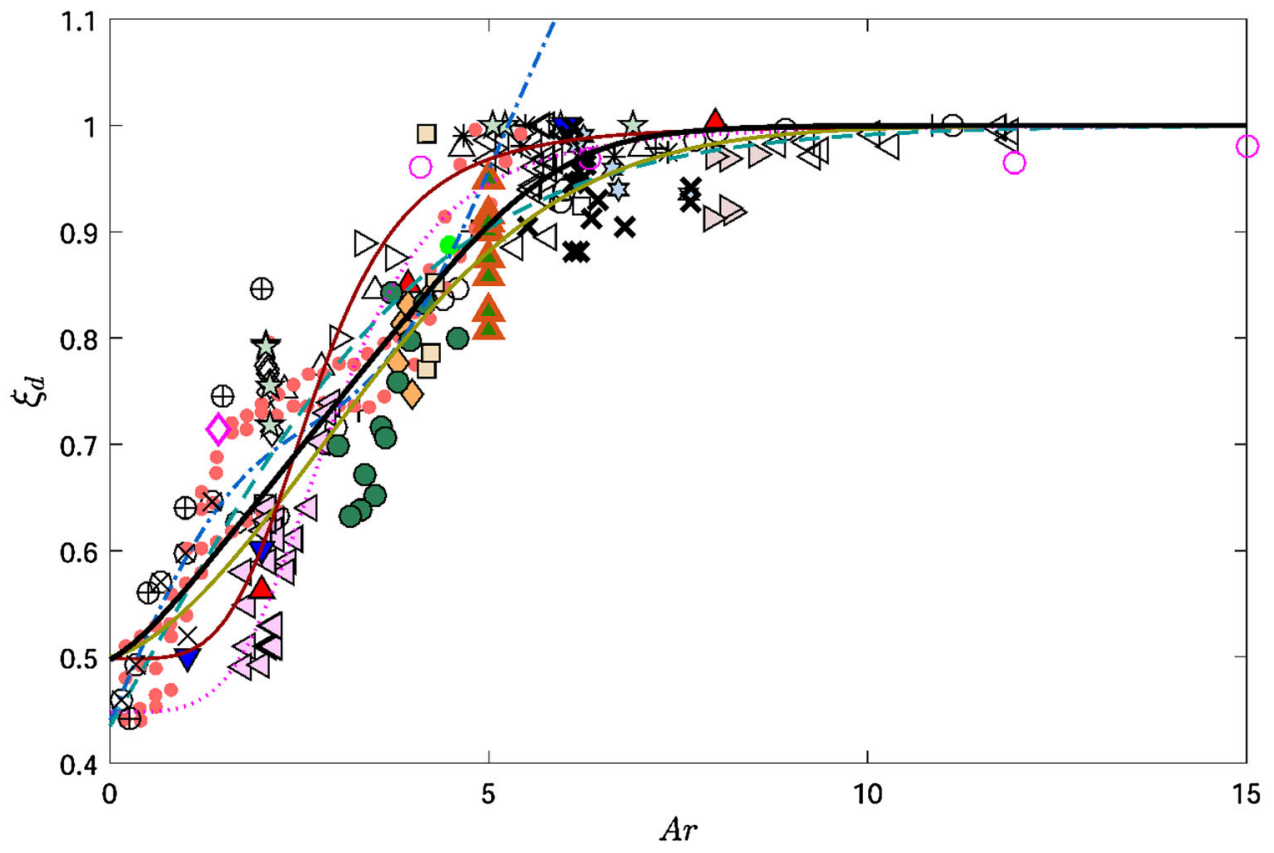

$\begin{array}{ll}\bigcirc & \text { Hu(1985) } \\ \square & \text { Sarma etal.(1983) } \\ \diamond & \text { Coleman(1986) } \\ \times & \text { Knight and Macdonald(1979) } \\ + & \text { Rajaram and Muralidhar(1969) } \\ \triangle & \text { Yan(2011) } \\ * & \text { Cardoso et al.(1989) } \\ \triangleleft & \text { Vanoni(1946) } \\ \triangleright & \text { Wang and Qian(1989) } \\ \otimes & \text { Murphy(1904) } \\ \bullet & \text { Nezu and Rodi(1986) }\end{array}$

$\begin{array}{ll}\oplus & \text { Girbson(1909) } \\ \square & \text { Wang and Fu(1991) } \\ \varangle & \text { Zippe and Graf(1983) } \\ \downarrow & \text { Kironoto(1993) } \\ \diamond & \text { Wang and An(1994) } \\ \triangleright & \text { Guy etal.(1996) } \\ \triangleleft & \text { Larrarte(2006) } \\ \nabla & \text { Nezu and Rodi(1985) } \\ \triangle & \text { Tominaga et al.(1989) } \\ \odot & \text { Song and Graf(1994) } \\ \times & \text { Montes and Ippen(1973) }\end{array}$

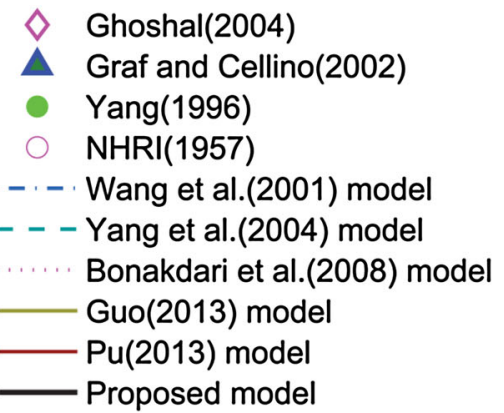

Fig. 4 Comparison with velocity dip position formula

computed accuracy results are shown in Table 2, suggesting that the proposed model has the best statistical results.

\section{Applications of the model}

The appropriate prediction of the velocity-dip-position is required to predict the Reynolds shear stress distribution and the vertical velocity distribution in open channels. This section explores the application of the proposed model and proposes its rational superiority compared to the other models.

\section{Prediction of total shear stress}

In turbulent flow through open channels, the total shear stress, composed of viscous shear stress and the Reynolds shear stress, is maximum at the channel bed and it gradually decreases linearly with vertical distance $y$ which is generally modeled as, $\tau_{\text {total }}=\mu \frac{\mathrm{d} u}{\mathrm{~d} y}-\rho \overline{u^{\prime} v^{\prime}}=\rho u_{*}^{2}\left(1-\frac{y}{h}\right)$

where $u^{\prime}$ and $v^{\prime}$ are fluctuation parts of the fluid velocities along longitudinal and vertical direction respectively, and $u_{*}$ is the shear velocity and $\rho$ is the fluid density. For wide open channels, the maximum velocity occurs at the free water surface and Eqs. (10) and (11) show that shear stress vanishes there. For a narrow channel, the maximum velocity at the central section always occurs below the water surface. In such a case the shear stress vanishes below the water surface where the maximum velocity occurs. Therefore, for narrow open channels, Eqs. (10) and (11) cannot be used as these do not satisfy the boundary condition $\frac{\overline{u^{\prime} v^{\prime}}}{u_{*}^{2}}=0$ at $\xi=\xi_{\mathrm{d}}$ and hence Eqs. (10) and (11) need to be modified. Yang et al. (2004) and Kundu (2015) proposed a modification to this equation as,

$$
\begin{aligned}
\tau_{\text {total }} & =\mu \frac{\mathrm{d} u}{\mathrm{~d} y}-\overline{\rho u^{\prime} v^{\prime}}=\rho u_{*}^{2}\left(1-\frac{y}{h}-\lambda \frac{y}{h}\right) \\
& =\rho u_{*}^{2}(1-\xi-\lambda \xi)
\end{aligned}
$$


Fig. 5 Prediction of total shear stress by Eqs. (10) and (11) using velocity-dip formulas

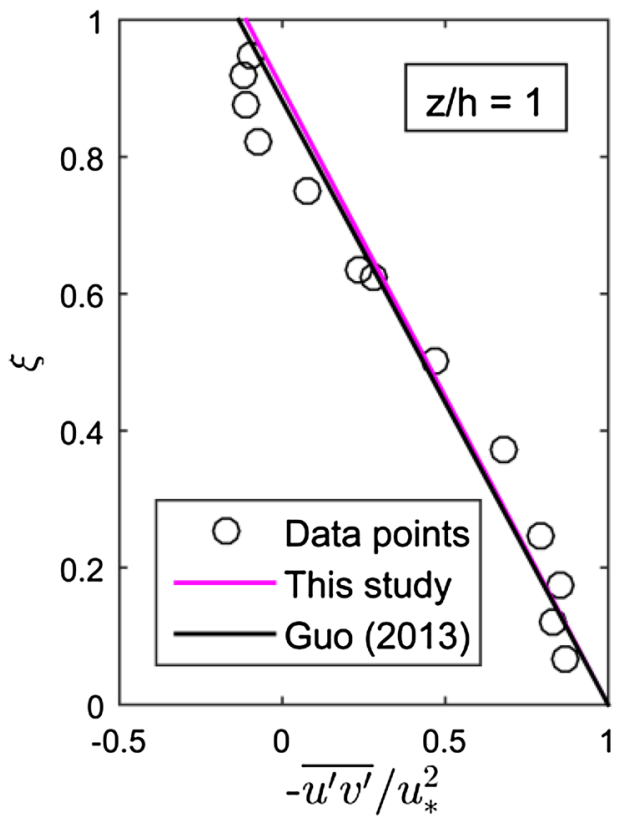

where $\lambda(>0)$ is the dip-correction parameter. They found that $\lambda$ changes with the aspect ratio of the channel as,

$\lambda=\frac{1}{\xi_{\mathrm{d}}}-1$

Kundu (2016) studied the effect of lateral roughness variation on the suspension profile. He analyzed the experimental data of Wang and Cheng (2006) and found that in wide open channels the presence of cellular secondary current affects the Reynolds shear stress which can be modeled as,

$\frac{\tau_{\text {total }}}{\rho u_{*}^{2}}=1-\xi-\beta \xi(1-\xi)$

where $\beta$ is a parameter whose value depends on location of dip-position. At two ends of a circular secondary cell, vertical velocity has opposite direction. It is well known that dip phenomenon occurs with the downward direction of the vertical velocity where $v<0$. Therefore, at one end of a circular secondary cell dip occurs, whereas on the other end vertical velocity $v>0$ and maximum velocity appear at the free surface. Accordingly, $\beta$ value can be taken as $\xi_{\mathrm{d}}$ over the length of a circular secondary cell.

In Fig. 5, experimental data of the total shear stress of Immamoto and Ishigaki (1988) are plotted together with the Eqs. (10) and (11). The experiment was carried out by Immamoto and Ishigaki (1988) using a laser Doppler anemometer, the aspect ratio, $b / h$ was 5 . In the figure the solid lines are plotted from Eqs. (10) and (11) where $\lambda$ is calculated from Eq. (12) using the present model of dip-position. From the figure it can observed that the proposed model provides good results for dipposition. It can be mentioned here that in the last figure, the model did not agree well with the data points. It happens due to the fluctuation of dip-position data points for $\mathrm{Ar} \leq 5$.

Figure 6 shows the validity of the proposed dip position model with the experimental data of Wang and Cheng (2006). The experiments were carried out in a straight rectangular tilting flume $18 \mathrm{~m}$ long, $0.6 \mathrm{~m}$ wide and $0.6 \mathrm{~m}$ deep. The bed comprised five rough and four smooth longitudinal strips, placed in an alternate manner. Each strip was $0.075 \mathrm{~m}$ in width except for two sidewall strips, which had half of the original width. The rough strips were prepared with densely packed fine gravel of uniform medium diameter of $2.55 \mathrm{~mm}$. The flow depth was $0.075 \mathrm{~m}$ and the aspect ratio Ar was maintained at 8 (wide open channel). The Reynolds shear stress is computed from Eq. (13) where $\beta$ is computed for each of the models of dip-position. The results in this figure show that the proposed model is effective for predicting the dip-position.

\section{Prediction of vertical velocity profile}

Several authors proposed models for vertical velocity distribution in sediment-laden open channel flows (Guo and Julien 2008; Yang et al. (2004); Bonakdari et al. (2008); Kundu and Ghoshal 2012). Kundu (2015) compared those models and found that the vertical velocity is best described by the model of Kundu and Ghoshal (2012) as: 
Fig. 6 Prediction of total shear stress by Eq. (13) using proposed dip formula
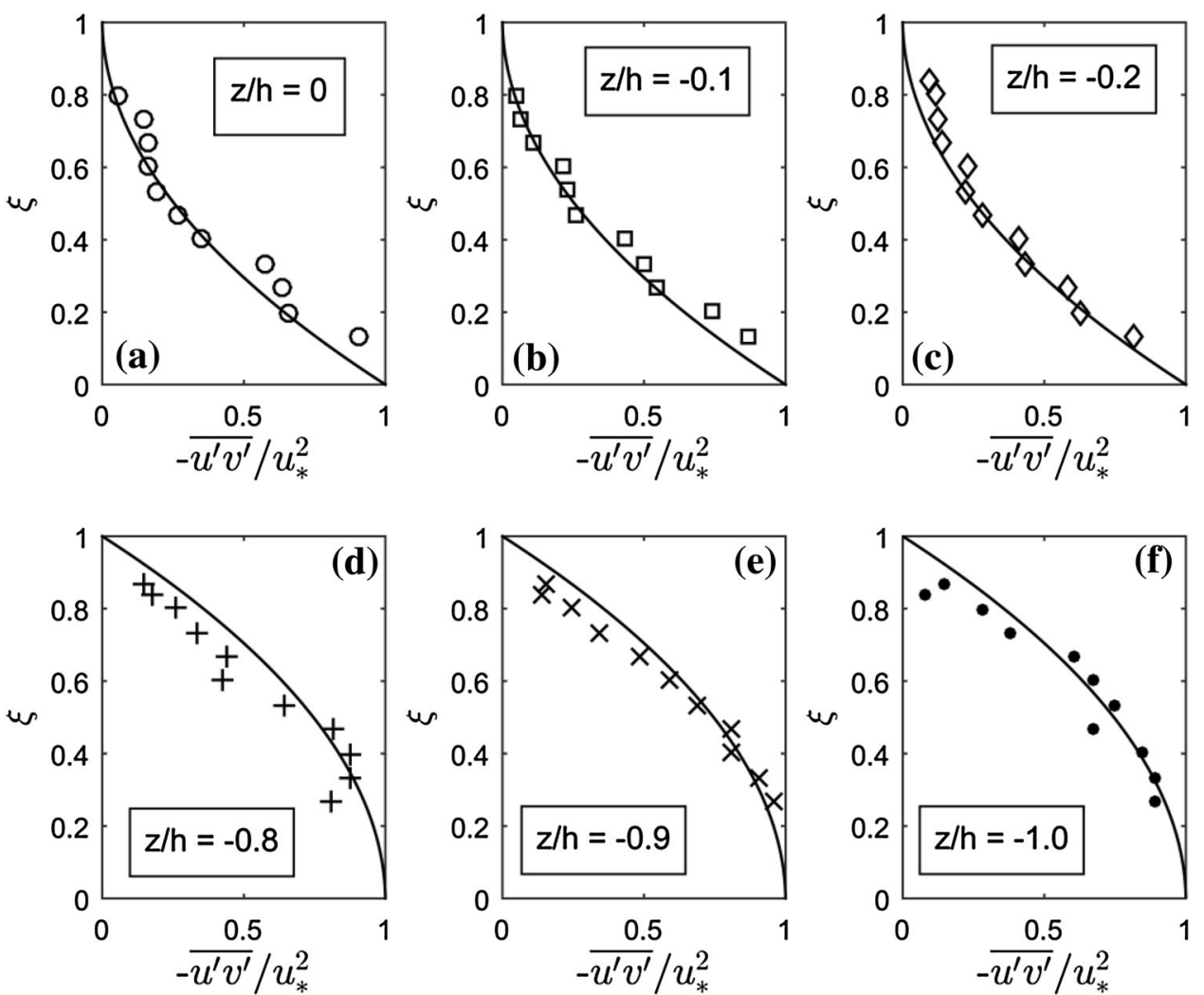

$\frac{u}{u_{*}}=\frac{1}{\kappa} \ln \left(\frac{\xi}{\xi_{0}}\right)+\frac{\lambda}{\kappa} \ln (1-\xi)+\frac{2 \Pi}{\kappa}\left(3 \xi^{2}-2 \xi^{3}\right)-\frac{4 \lambda \Pi}{\kappa} \xi^{3}$

where $\xi_{0}$ is the distance from the bed at which the velocity is hypothetically equal to zero, $\kappa=0.41$ is the von Karman coefficient, $\Pi$ is the Cole's wake parameter and $\lambda$ is the dip correction parameter computed from Eq. (12). Equation (14) predicts the velocity profile if the parameters $\Pi$ and $\lambda$ are correctly chosen. The value of $\Pi$ is taken from experimental data. The value of $\lambda$ is calculated from Eq. (12) for all the models of dip-position.

Figure 7 shows the comparison of velocity profiles computed from Eq. (14) with value of $\lambda$ from Eq. (12) for the experimental data of Coleman (1986). Coleman (1986) did experiments in a smooth flume which was $356 \mathrm{~mm}$ wide and $15 \mathrm{~m}$ long. During the experiments, the energy slope was kept to be 0.002. Among 40 test cases, test cases 1, 21, and 32 were performed in clear water flow. In the experiment, the location of dip-position was also obtained. Figure 7 shows the result for RUN 1 of Coleman (1986) data. The value of $\Pi$ is computed as follows: initially the value of $\xi_{\mathrm{d}}$ is taken from experimental data set of Coleman (1986), and then the value of $\lambda$ is calculated from Eq. (12). After that the value of $\Pi$ is computed by using the least squares method using experimental data. The value of $\Pi$ thus obtained is kept fixed for all dip-position models. It can be observed from the figure that when value of $\lambda$ is computed from model of Wang et al. (2001) and Yang et al. (2004), it overestimate the maximum velocity and model of Bonakdari et al. (2008) underestimates the maximum velocity. Whereas the proposed model, and models of Guo (2013) and $\mathrm{Pu}$ (2013) give satisfactory results. To get a quantitative result, the RMSE error is computed for all the models which are shown in Table 3. Table shows that the velocity model gives the best result when parameter $\lambda$ is computed from the model proposed in this study.

\section{Conclusions}

The velocity dip position model proposed in this study has been fitted by asymptotic matching technique with data obtained from a wide range of aspect ratios for open channels of both smooth and rough bed surfaces. The employed fitting asymptotic technique has allowed matching the lower and upper boundaries of dip positions equal to 0.5 and 1 , respectively. The comparison performed with other formulas showed that the proposed model provides the best-fit accuracy results. It is thus advocated here that the proposed model is capable of predicting velocity dip position in open channels under different conditions of aspect ratio and bed roughness. Also, the application results show that the proposed model provides better 

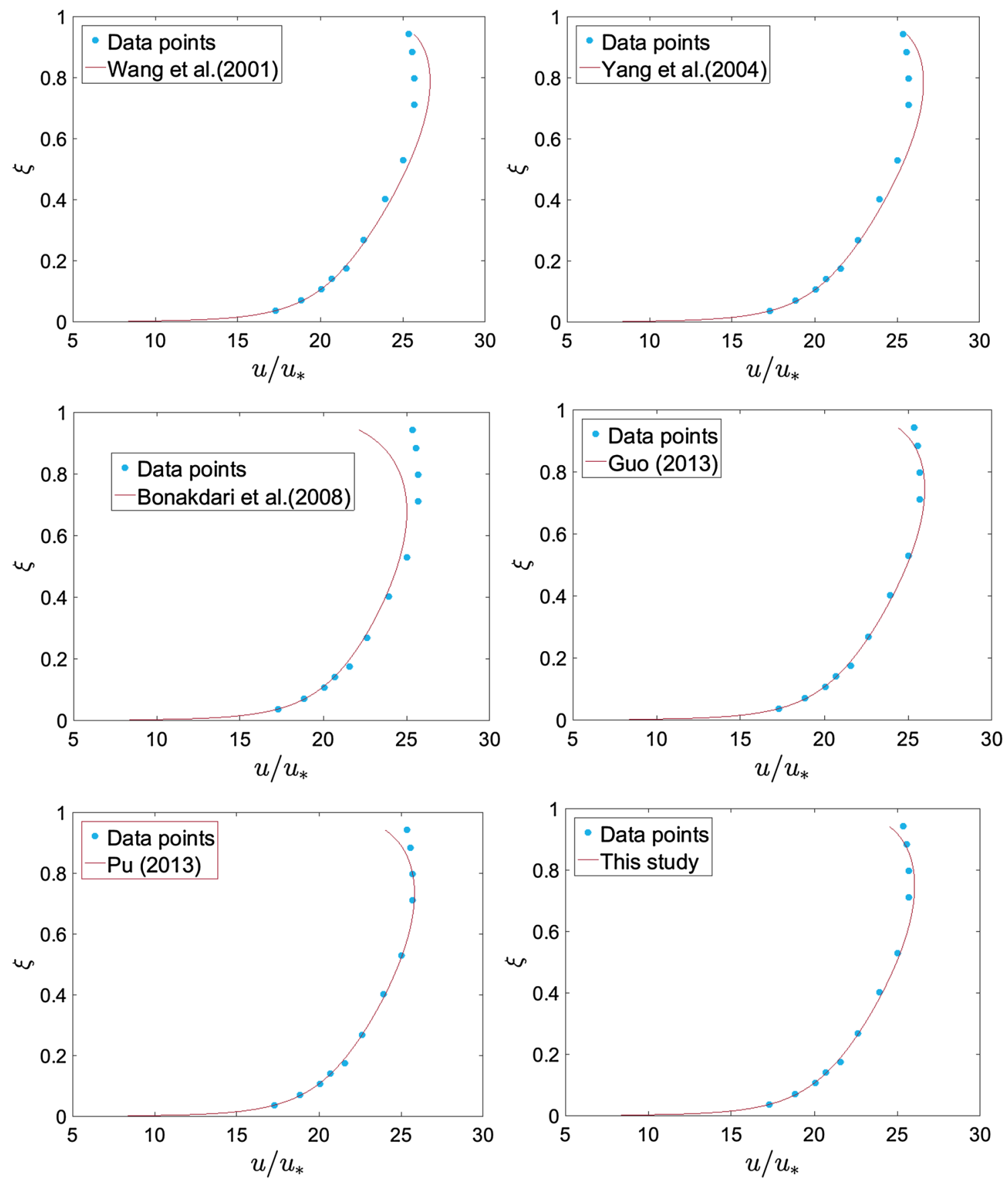

Fig. 7 Prediction of mean velocity by Eq. (14) using dip formulas

Table 3 Accuracy of the model for predicting velocity profile

\begin{tabular}{lllllll}
\hline Coleman (1986) runs & Wang et al. (2001) & Yang et al. (2004) & Bonakdari et al. (2008) & Guo (2013) & Pu (2013) & This study \\
\hline 1 & 0.4781 & 0.4417 & 1.1527 & 0.3134 & 0.4172 & $0.2926^{*}$ \\
21 & 0.5273 & 0.5060 & 1.1681 & 0.3956 & 0.4608 & $0.3765^{*}$ \\
32 & 0.4962 & 0.4551 & 1.0898 & 0.2788 & 0.3787 & $0.2640^{*}$ \\
\hline
\end{tabular}

* denotes minimum error 
parameter estimation than the other models in terms of computing the Reynolds shear stress and mean velocity distribution over the full water depth of open channels.

Acknowledgements The author is thankful to Dr. Jaber Almedeij at Kuwait University, Kuwait for his help and fruitful comments. Author is also thankful to all the reviewers for their helpful and constructive comments.

Open Access This article is distributed under the terms of the Creative Commons Attribution 4.0 International License (http:// creativecommons.org/licenses/by/4.0/), which permits unrestricted use, distribution, and reproduction in any medium, provided you give appropriate credit to the original author(s) and the source, provide a link to the Creative Commons license, and indicate if changes were made.

\section{References}

Almedeij J (2008) Drag coefficient of flow around a sphere: matching asymptotically the wide trend. Powder Technol 186(3):218-223

Almedeij J (2009) Asymptotic matching with a case study from hydraulic engineering. In: Recent advances in water resources, hydraulics and hydrology. Cambridge, pp 71-76

Almedeij J (2010) Modeling shields diagram by asymptotic matching. Kuwait J Sci Eng 37(2B):43-58

Bonakdari H, Larrarte F, Lassabatere L, Joannis C (2008) Turbulent velocity profile in fully-developed open channel flows. Environ Fluid Mech 8(1):1-17

Cardoso AH, Graf WH, Gust G (1989) Uniform flow in smooth openchannel. J Hydraul Res 27(5):603-616

Coleman NL (1986) Effects of suspended sediment on the openchannel velocity distribution. Water Resour Res 22(10): 1377-1384

Einstein HA (1950) The bed load function for sediment transportation in open channels. Technical bulletin 1026. Soil Conservation Service, USDA, Washington, DC

Francis JB (1878) On the cause of the maximum velocity of water flowing in open channels being below the surface. Trans Am Soc Civil Eng 7(1):109-113

Ghoshal K (2004) On velocity and suspension concentration in a sediment-laden flow: experimental and theoretical studies. Ph.D. thesis, Indian Statistical Institute, Kolkata

Gibson AH (1909) On the depression of the filament of maximum velocity in a stream flowing through an open channel. Proc $\mathrm{R}$ Soc A Math Phys Sci 82:149-159

Graf WH, Cellino M (2002) Suspension flows in open channels: experimental study. J Hydraul Res 40(4):435-447

Guo J (2013) Modified log-wake-law for smooth rectangular open channel flow. J Hydraul Res 52(1):121-128

Guo J, Julien PY (2008) Application of the modified log-wake law in open-channels. J Appl Fluid Mech 1(2):17-23

Guo J, Mohebbi A, Zhai Y, Clark SP (2015) Turbulent velocity distribution with dip phenomenon in conic open channels. J Hydraul Res 53(1):73-82

Guy HP, Simons DB, Richardson EV (1966) Summary of alluvial channel data from flume experiments, 1956-1961. Tech. rep., United States geological survey water supply paper number 462-1, Washington DC

$\mathrm{Hu} \mathrm{CH}$ (1985) Effects of width-to-depth ratio and side wall roughness on velocity distribution and friction factor. MS thesis. Tsinghua University, Beijing, China (in Chinese)
Hu C, Hui Y (1995) Mechanical and statistical laws of open channel sediment-laden flow. Science Press, Beijing (in Chinese)

Immamoto H, Ishigaki T (1988) Measurement of secondary flow in an open channel. In: Proc. 6th IAHR-APD Congress, pp 513-520

Kironoto BA, Graf WH (1994) Turbulence characteristics in rough uniform open-channel flow. Proc ICE Water Marit Energy 106(12):333-344

Knight DW, Macdonald JA (1979) Open-channel flow with varying bed roughness. J Hydraul Div 105(9):1167-1183

Kundu S (2015) Theoretical study on velocity and suspension concentration in turbulent flow. Ph.D. thesis, IIT Kharagpur, Kharagpur

Kundu S (2016) Effect of lateral bed roughness variation on particle suspension in open channels. Environ Earth Sci. doi:10.1007/ s12665-016-5418-7

Kundu S (2017) Prediction of velocity-dip-position over entire cross section of open-channel flows using entropy theory. Environ Earth Sci. doi:10.1007/s12665-017-6695-5

Kundu S, Ghoshal K (2012) An analytical model for velocity distribution and dip-phenomenon in uniform open channel flows. Int J Fluid Mech Res 39(5):381-395

Larrarte F (2006) Velocity fields in sewers: an experimental study. Flow Meas Instrum 17:282-290

Lee JS, Julien PY (2006) Electromagnetic wave surface velocimetry. J Hydraul Eng ASCE 132(2):146-153

Montes JS, Ippen AT (1973) Introduction of two-dimensional turbulent flow with suspended particles. Report of Ralph M. Parsons Laboratory Paper No. 164, Massachusetts Institute of Technology, Cambridge

Murphy C (1904) Accuracy of stream measurements. Water Supply Irrig Paper 95:111-112

Nezu I, Nakagawa H (1993) Turbulence in open-channel flows. IAHR Monograph, Balkema, Rotterdam

Nezu I, Rodi W (1985) Experimental study on secondary currents in open channel flow. 21th IAHR Congress. IAHR, Melbourne, pp 115-119

Nezu I, Rodi W (1986) Open-channel flow measurements with a laser dropper anemometer. J Hydraul Eng 112(5):335-355

NHRI (1957) Experimental study on 3D velocity distribution in smooth flow. National Historic Research Institute (NHRI), Nanjing Hydraulic Research Institute, Nanjing

Pu JH (2013) Universal velocity distribution for smooth and rough open channel flows. J Appl Fluid Mech 6(3):413-423

Rajaratnam N, Muralidhar D (1969) Boundary shear stress distribution in rectangular open channels. La Houille Blanche 24(6):603-609

Sarma KVN, Lakshminarayana P, Rao NSL (1983) Velocity distribution in smooth rectangular open channels. J Hydraul Eng 109:270-289

Song TC, Graf WH (1994) Non-uniform open channel flow over a rough bed. J Hydrosci Hydraul Eng 12(1):1-25

Stearns FP (1883) On the current-meter: together with a reason why the maximum velocity of water flowing in open channel is below the surface. Trans Am Soc Civil Eng 12(1):301-338

Tominaga A, Nezu I, Ezaki K, Nakagawa H (1989) Three dimensional turbulent structure in straight open channel flows. J Hydraul Res 27(1):149-173

Vanoni VA (1946) Transportation of suspended sediment by running water. Trans ASCE 111:67-133

Wang X, An F (1994) The fluctuating characteristics of hydrodynamic forces on bed particles. Int J Sediment Res 9(3):183-192

Wang ZQ, Cheng NS (2006) Time-mean structure of secondary flows in open channels with longitudinal bedforms. Adv Water Resour 29:1634-1649

Wang X, Fu R (1991) Study on the velocity profile equations of suspension flows. In: 24th IAHR Congress, Madrid. pp C3-C10

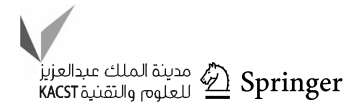


Wang X, Qian N (1989) Turbulence characteristics of sediment-laden flows. J Hydraul Eng 115(6):781-799

Wang X, Wang Z, Yu M, Li D (2001) Velocity profile of sediment suspensions and comparison of log-law and wake-law. J Hydraul Res 39(2):211-217

Yan J, Tang H, Xia Y, Li K, Tian Z (2011) Experimental study on influence of boundary on location of maximum velocity in open channel flows. Water Sci Eng 4(2):185-191

Yang SQ (1996) Interactions of boundary shear stress, velocity distribution and flow resistance in 3d open channels. Ph.D. thesis, Nanyang Technological University, Singapore
Yang SQ, Tan SK, Lim SY (2004) Velocity distribution and dipphenomenon in smooth uniform open channel flows. J Hydraul Eng 130(12):1179-1186

Zippe HJ, Graf WH (1983) Turbulent boundary-layer flow over permeable and non-permeable rough surfaces. J Hydraul Res 21(1):51-65 